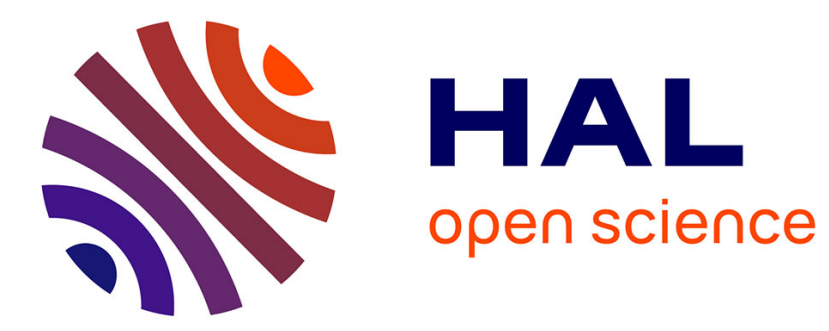

\title{
Les Evaluations Sociales en Stratégie : Légitimité, Réputation, Statut, Stigmate et Cie.
}

Thomas Roulet

\section{To cite this version:}

Thomas Roulet. Les Evaluations Sociales en Stratégie : Légitimité, Réputation, Statut, Stigmate et Cie.. Les grands courants en management stratégique., A paraître. hal-01970557

\section{HAL Id: hal-01970557 \\ https://hal.science/hal-01970557}

Submitted on 5 Jan 2019

HAL is a multi-disciplinary open access archive for the deposit and dissemination of scientific research documents, whether they are published or not. The documents may come from teaching and research institutions in France or abroad, or from public or private research centers.
L'archive ouverte pluridisciplinaire HAL, est destinée au dépôt et à la diffusion de documents scientifiques de niveau recherche, publiés ou non, émanant des établissements d'enseignement et de recherche français ou étrangers, des laboratoires publics ou privés. 


\title{
Les Evaluations Sociales en Stratégie : Légitimité, Réputation, Statut, Stigmate et Cie.
}

\author{
Thomas Roulet \\ University of Cambridge
}

A paraître dans «Les grands courants en management stratégique. »S. Liarte, Editions EMS

\begin{abstract}
:
Ce chapitre se focalise sur les évaluations sociales des organisations - les cadres conceptuels qui permettent de comprendre la façon dont les organisations sont perçues et comment cela affecte en retour leurs relations avec les parties prenantes. Nous explorons les différences entre évaluations sociales positives - légitimité, statut et réputation - et négatives telles le stigmate ou la désapprobation. Au-delà du niveau organisationnel, ces concepts ont été analysés à d'autres niveaux avec des travaux se focalisant sur les groupes ou les individus. Nous discutons ensuite des implications méthodologiques de l'étude des évaluations sociales, ainsi que des pistes futures de recherche dans ce domaine.
\end{abstract}

Comment les organisations et les individus sont-ils perçus ? Pourquoi ? Et comment ces perceptions affectent-elles la vie sociale et économique de ces acteurs évalués et évaluateurs ? La question des évaluations sociales en sciences de gestion, inspirée en partie par les travaux de disciplines connexes en sociologie (Lamont, 2012) ou psychologie (Glass et Ellett, 1980), a trouvé écho dans un large spectre de recherches tant théoriques qu'empiriques. Cette thématique a donné naissance à une foison de concepts, qui se distinguent mais aussi parfois se superposent et se révèlent difficiles à distinguer. La légitimité, la réputation ou le statut sont parmi les évaluations sociales les plus communément examinées en stratégie (Philippe, 2009). Ainsi, l'impact de la réputation sur la performance, bien qu'ayant d'abord intéressé les chercheurs en finance (Carter et Manaster, 1990) a été l'un des questionnements empiriques clefs en stratégie (Roberts et Dowlings, 2002). De la même manière, le statut organisationnel est principalement connu pour son application a la finance (Podolny, 1993), mais depuis a été utilisé dans de très nombreux contextes et pour expliquer une variété de 
phénomènes en stratégie et théorie des organisations (Piazza et Castellucci, 2014). Enfin, la légitimité fait l'objet d'une très vaste littérature notamment de par son intersection avec la théorie néo-institutionnelle (Suchman, 1995 ; Deephouse et Suchman, 2008) et la réflexion plus vaste que les sciences de gestion ont autour de la conformité des organisations aux normes sociales et sociétales (Philippe, 2006 ; Reynaud et Walas, 2015 ; Roulet, 2015b). D'autres concepts comme la célébrité (Zavyalova, et al., 2017), l'évaluation par les pairs (Shymko et Roulet, 2017), l'identité de marché (Wang, Wezel et Forgues, 2015) ont encore ajouté un niveau de complexité à ce champ émergent.

En parallèle, ce sont développés les travaux sur les évaluations sociales négatives, faisant l'hypothèse que les évaluations sociales négatives et positives sont sur deux continua différents (Hudson, 2008). La présence d'une multiplicité d'audiences peut, en effet, permettre à une organisation d'être simultanément positivement et négativement perçue (Roulet, 2015b). Parmi les évaluations sociales négatives, la plus importante est le stigmate organisationnel (Hudson, 2008 ; Roulet, 2015a) mais d'autres notions plus proches de leur forme d'opérationnalisation ont été utilisées dans la littérature. On notera ainsi les conceptualisations de la désapprobation publique (Vergne, 2012 ; Clemente et Roulet, 2015), la couverture media négative (Westphal et Deephouse, 2011 ; Zavyalova et al., 2016), ou encore de la réputation négative (Mishina et Devers, 2012). Ces concepts, ayant émergé plus récemment que leurs pendants positifs, ont permis de renforcer la conceptualisation de phénomènes contemporains dans le monde de l'entreprise tels que les scandales (Adut, 2008 ; Daudigeos, Roulet et Valiorgue,. A paraître) ou des comportements illicites (Palmer, 2012).

Les effets des évaluations sociales sur la performance sont établis empiriquement (Roberts et Dowling, 2002) malgré la nature parfois récursive et endogène de cette relation (Deephouse et Carter, 2005). Les évaluations sociales qu'elles soient positives ou négatives jouent aussi un rôle crucial dans la façon dont les entreprises sont sélectionnées par leurs clients (Carter et Manaster, 1990), par les banquiers (Cusin et Maymo, 2017), mais aussi dans l'identification par les parties prenantes (Zavyalova, et al., 2016) en particulier les employés (Roulet, 2017), et la diffusion des pratiques (Clemente et Roulet, 2015). Mais de plus en plus, les évaluations sociales sont examinées par les chercheurs en stratégie comme la «variable dépendante », c'est-à-dire une mesure de performance non financière de l'entreprise (par exemple Philippe, 2006 ; 2009 ; Vergne, 2012 ; Wang, Wezel et Forgues, 2015 ; Reynaud et Walas, 2015). L'évaluation sociale des organisations peut être clef dans certaines industries comme par exemple les industries culturelles dont la survie dépend plus de leur 
reconnaissance (Shymko et Roulet, 2017) ou de leur visibilité (Elsbach et Sutton, 1992 ; Helms et Patterson, 2014).

Les défis à relever pour qui souhaite mobiliser cette littérature et ces concepts sont cependant nombreux. Tout d'abord, les différents concepts sont particulièrement difficiles à différencier (comme souligné par Deephouse et Carter, 2005 ; Barnett, et al., 2006 ; Devers, et al., 2009), ce qui est en particulier lié aux difficultés de l'opérationnalisation. Ainsi nous commençons ce chapitre par un retour aux définitions. Au-delà de la conceptualisation, les évaluations sociales étant des notions isolées plutôt que des théories à part entière, la compréhension de ces notions a reposé sur un jeu de cadres conceptuels allant de la théorie des ressources (Deephouse, 2000) à la théorie institutionnelle (Suchman, 1995 ; Bitektine, 2011) en passant plus récemment par les travaux sur les catégories (Paolella et Durand, 2016). Le traitement des évaluations sociales nécessite donc une certaine polyvalence et connaissance étendue des cadres théoriques. Dans cette revue de littérature, nous présentons les éléments clefs de la théorisation des évaluations sociales : le rôle des audiences et des médias. Enfin, de par leur nature abstraite et parfois difficile à capturer empiriquement, les défis méthodologiques sont nombreux. Nous mentionnons les différentes approches méthodologiques qui peuvent être utiles pour explorer les dynamiques d'évaluation sociale. Enfin, nous finissons par traiter les perspectives et opportunités que ce champ continue d'offrir.

\section{DEFINITION DES DIFFERENTES FORMES D' EVALUATION SOCIALE}

Le champ des évaluations sociales a emprunté a de nombreuses disciplines au-delà des sciences de gestion. La sociologie offre une très vaste littérature où évaluation et valorisation sont des questions intimement liées (Lamont, 2012). Les travaux de Podolny sur les banques d'investissement ont, par exemple, fait le pont entre la sociologie et le management (Podolny, 2010). Bien que la psychologie se soit penchée principalement sur le niveau individuel (Glass et Ellett, 1980), statut et réputation sont des concepts clefs dans ce champ (voir par exemple Anicich, et al., 2015). La question des évaluations sociales était déjà au centre du concept de représentation mentale de l'Ecole des Annales en histoire (Clemente, Durand et Roulet, 2017).

Le foisonnement des concepts a rendu de plus en plus difficile la distinction entre ces différentes notions, c'est pourquoi une clarification des définitions s'impose. Nous nous 
focalisons sur les principales formes d'évaluation sociale mais notons aussi les autres concepts connexes. Dans cette section, nous faisons aussi la différence entre évaluation sociale positive et négative. Enfin, parce que les évaluations sociales se formulent non seulement au niveau des organisations mais à tous les niveaux d'analyse - pratiques, individus, champs et industries -, nous expliquons les liens entre ces différents niveaux d'analyse et comment ils peuvent aider à conceptualiser et comprendre les évaluations sociales des organisations, leurs antécédents et conséquences.

\subsection{Evaluations sociales positives}

Dans leurs essais théoriques, Devers et al. (2009) ainsi que Bitektine (2011) tentent de différencier ces trois notions, malgré la superposition des concepts et la confusion qui peut exister dans la façon dont ils sont définis. De manière générale, la légitimité traduit la conformité aux normes sociales, et donc l'acceptation sociale de l'organisation (Roulet, 2015b). Etre responsable socialement fournit, par exemple, de la légitimité aux organisations (Reynaud et Walas, 2015). De son côté, la réputation d'une organisation est un signal de la qualité du service que peut fournir l'organisation (Philippe, 2009) bien que certains travaux définissent la réputation comme un signe d'acceptation publique qui s'avère proche de la définition de la légitimité (voir par exemple McDonnell et King, 2013 ; Zavyalova et al., 2016). Les deux approches sont cependant réconciliables si l'on considère que dans ces deux travaux empiriques, ce qui est évalué est la façon dont l'organisation rend service à la société plutôt qu'à une audience plus restreinte comme par exemple les clients (Carter et Manaster, 1990 ; Bitektine, 2011). Certains travaux vont jusqu'à distinguer la réputation de l'organisation pour chacune de ses audiences - experts, clients, pairs (Boutinot et al., 2017). Inversement, en ce qui concerne la légitimité, les piliers cognitif (l'existence de l'organisation est considérée comme acquise) et pragmatique (l'organisation répond de manière satisfaisante aux demandes des parties prenantes qui l'évaluent) de ce concept sont susceptibles d'être associés à (ou confondus avec) la réputation (Deephouse et Carter, 2005 ; Suddaby, et al., 2017). Bitektine (2011) identifie plus de 17 formes de légitimité mais reconnait la primauté des piliers cognitifs, pragmatiques et normatifs (le degré de conformité aux normes sociales).

Le statut, quant à lui, est une position au sein d'une hiérarchie établie et acceptée par les parties prenantes (Podolny, 2010; Piazza et Castellucci, 2015). Le statut peut, par exemple, se traduire par l'ordre dans lequel les banques d'investissement sont citées dans les documents d'émissions de produits financiers (Podolny, 1993). La question du statut est aussi liée à celle du pouvoir des organisations les unes sur les autres, et de leur dépendances 
mutuelles (Chiambaretto, 2015) ainsi que de leur position au sein de réseaux sociaux (Washington et Zajac, 2005). La revue de littérature par Piazza et Castellucci (2015) différencie le statut au sein de marché (niveau macro), le statut dans des environnements formalisés comme les organisations (niveau meso), et le statut au sein de groupes et équipes (niveau micro).

Le tableau 1 résume les principales évaluations sociales (légitimité, réputation et statut), leurs définitions et les principaux travaux sur la question.

Tableau 1 - Légitimité, réputation et statut - Sélection de travaux existants

\begin{tabular}{|l|l|}
\hline Concept & Liste non exhaustive de travaux marquants \\
\hline $\begin{array}{l}\text { Légitimité organisationnelle } \\
\text { Définition : La légitimité organisationnelle } \\
\text { revêt la conformité aux normes, et } \\
\text { l'acceptation sociale de l'organisation } \\
\text { (Suchman, 1995; Roulet, 2015b) }\end{array}$ & $\begin{array}{l}\text { Conceptualisation : Dowling et Pfeffer (1975); } \\
\text { Suchman (1995); Bitektine (2011) } \\
\text { Exploration empirique : Philippe (2006), Reynaud } \\
\text { et Walas (2015), Roulet (2015b) } \\
\text { Revue de la littérature : Deephouse et Suchman } \\
(2008) \text {; Suddaby et al. (2017). }\end{array}$ \\
\hline $\begin{array}{l}\text { Réputation organisationnelle } \\
\text { Définition : Signal de la qualité du service ou } \\
\text { produit offert par l'organisation (Fombrun et } \\
\text { Shanley, 1990; Barnett et al., 2006) }\end{array}$ & $\begin{array}{l}\text { Conceptualisation : Barnett et al. (2006), Rhee et } \\
\text { Exploration empirique : Fombrun et Shanley } \\
\text { (1990), Roberts et Dowling (2002), Gaultier- }\end{array}$ \\
$\begin{array}{l}\text { Gaillard et Pratlong (2011), Philippe et Durand } \\
(2011) \\
\text { Revue de la littérature : Walker (2010) }\end{array}$ \\
\hline $\begin{array}{l}\text { Statut organisationnel } \\
\text { Définition : Position sociale dans une } \\
\text { hiérarchie établie et reconnue (Piazza et } \\
\text { Castellucci, 2014) }\end{array}$ & $\begin{array}{l}\text { Conceptualisation : Podolny (2010) } \\
\text { Exploration empirique : Podolny (1993), } \\
\text { Benjamin et Podolny, (1999). Washington et Zajac } \\
(2005), \text { Collet et Philippe (2014) } \\
\text { Revue de la littérature : Piazza et Castellucci } \\
\text { (2015) }\end{array}$ \\
\hline
\end{tabular}

Au-delà de ces trois formes d'évaluation sociale, on notera aussi un certain nombre d'autres travaux qui s'appuient sur différentes conceptualisations. Parmi ces concepts, on peut lister entre autres l'image organisationnelle (Dutton et Dukerich, 1991), la célébrité (Zavyalova, et al., 2017), l'évaluation par les pairs (Shymko et Roulet, 2017), la renommée (Boutinot et al., 2017), ou encore l'identité organisationnelle ou de marché (Wang et al., 2015). L'image et l'identité organisationnelle sont souvent confondus avec la réputation (Barnett et al., 2006), comme celle-ci a souvent des attributs en commun avec la légitimité (Philippe, 2006). Ces concepts sont cependant liés : Barnett et al. (2006) considèrent, par exemple, l'image organisationnelle comme un antécédent de la réputation en présentant cette 
dernière comme un sous-ensemble d'évaluation par les parties prenantes ayant un impact sur la performance de l'organisation.

Le concept d'identité de marché reflète les évaluations du positionnement compétitif des organisations par des acteurs externes (Wang, et al., 2015) contrairement à l'identité organisationnelle qui est une évaluation de l'organisations fournie par les acteurs internes (Elsbach et Kramer, 1996). L'identité organisationnelle ou l'identité de marché font toutes deux le lien avec les évaluations sociales négatives. Wang et al. (2015) observe, par exemple, la façon dont les hôtels sont affectés par les critiques des clients sur TripAdvisor. Elsbach et Kramer (1996) confirment la menace que font peser les évaluations sociales négatives pour l'identité organisationnelle. Roulet (2015b) établit un lien entre les évaluations négatives des médias extérieurs aux champs et les évaluations positives des acteurs à l'intérieur du champ. De la même manière, la désapprobation d'une organisation peut renforcer l'identification des employés par effet de contraste (Roulet, 2017).

Etre positivement évalué a généralement de nombreuses conséquences tangibles et favorables. Le statut, la réputation et la légitimité et autres évaluations positives rendent les entreprises plus attractives pour les clients potentiels (Podolny, 1993), pour les acteurs de champ (Piazza et Castellucci, 2015) et pour les employés potentiels (Turban et Greening, 1997) ou actuels (Roulet, 2017). De manière plus complexe, l'image de l'organisation influence sa trajectoire en guidant la façon dont ses membres interprètent les données de leur environnement et agissent en conséquence (Dutton et Dukerich, 1991). Au final, ces évaluations positives rendent l'organisation plus performante (Deephouse, 2000 ; Deephouse et Carter, 2005). Comme on pourrait s'y attendre de par la différence entre les deux concepts, la performance a un effet positif sur la réputation, mais pas forcément sur la légitimité (Deephouse et Carter, 2005), puisque celle-ci dépend plus de la conformité à des normes au niveau sociétal. Les organisations possédant un statut élevé bénéficient de privilèges, en étant par exemple inviter à participer à des échanges inter-organisationnels plus prestigieux (Washington et Zajac, 2005). De manière générale, les organisations possédant un statut élevé peuvent tirer parti plus aisément de leur collaboration en usant de cet avantage pour demander plus de leurs partenaires, ce qui compense le désavantage que représente le fait de s'associer avec une organisation de statut moins élevé (Castellucci et Ertug, 2010). La réputation et le statut ont des effets indépendants et positifs sur le prix des produits vendus (Benjamin et Podolny, 1999) mais d'autres travaux ont trouvé des effets légèrement divergents : par exemple, la réputation d'un fournisseur de ressources influence les revenus du récipient via 
l'effet médiateur de la qualité du produit tandis que l'effet du statut est plus direct (Ertug et Castellucci, 2013). Cette différence est principalement due au fait qu'avoir un fournisseur avec un statut élevé agit comme un signe d'approbation extérieur et donc plus intangible, alors que la réputation signale une qualité tangible de service ou de produit qui se traduit directement par une performance plus élevée. Mais les évaluations sociales positives sont souvent traitées comme un capital que l'on peut perdre, en parlant par exemple de risque réputationnel (Gaultier-Gaillard et Pratlong, 2011). Mais comme Hudson (2008) le suggère, l'absence de légitimité diffère de l'illégitimité et c'est pourquoi il est important de distinguer deux continua selon la nature des évaluations sociales qu'elles soient positives ou négatives.

\subsection{Evaluations sociales négatives}

Les évaluations sociales négatives font l'objet d'une littérature beaucoup moins structurée mais toute aussi foisonnante. Si les travaux sur l'illégitimité se confondent souvent avec les travaux sur la légitimité (Bitektine, 2011), Hudson (2008) suggère l'existence de deux continua différents et potentiellement divergents pour traiter les évaluations sociales négatives et positives : il insiste sur le fait qu'elles ne sont pas forcément opposées et même parfois concomitantes. L’illégitimité pour une audience peut même être la conséquence de la légitimité pour une autre audience (Roulet, 2015b).

L'évaluation sociale négative la plus documentée est le stigmate organisationnel (Hudson, 2008; Roulet, 2015a) qui se fonde sur une littérature riche et qui continue de foisonner au niveau individuel dans le champ de la sociologie (Goffman, 1963 ; Link et Phelan, 2001 ; Stenger et Roulet, 2017). Une organisation stigmatisée est une organisation dont l'image est entachée du point de vue collectif des parties prenantes (Devers, et al., 2009). Les travaux sur le stigmate organisationnel font la différence entre les organisations qui subissent un stigmate de par leur nature et leur seule existence («core-stigma») (Wolfe et Blithe, 2015) ou un stigmate épisodique (Hudson, 2008). Les industries stigmatisées incluent des organisations du premier groupe - l'appartenance à une industrie contestée de par ses activités communiquent à ses membres un stigmate par nature (Galvin et al. 2005). Les entreprises dont l'activité est répréhensible ou moralement condamnable font, de manière générale, partie de cette catégorie (Hudson et Okhuysen, 2009) : par exemple, l'industrie de l'armement (Vergne, 2012), les saunas (Hudson et Okhuysen, 2009), les maisons closes (Wolfe et Blithe, 2015) ou le nucléaire (Piazza et Perretti, 2015). Le stigmate épisodique, $a$ contrario, est le plus souvent dû a un écart de conduite ponctuel générant un scandale 
(Hudson, 2008). D'autres travaux identifient la désapprobation comme la variance au sein d'une catégorie d'organisations stigmatisées utilisant ainsi le concept de catégorisation que nous expliquons ci-dessous (Vergne, 2012; Piazza et Perretti, 2015). Enfin, Mishina et Devers (2012) différencient mauvaise réputation et stigmate : ils soulignent le fait que la réputation varie tandis que le stigmate signale une appartenance dichotomique (une vision proche du processus de catégorisation théorisée par Vergne (2012)). Une mauvaise réputation est une évaluation signalée tandis que le stigmate est le résultat d'un processus de labellisation par les parties prenantes. Avoir «mauvaise presse », soit une mauvaise réputation signalée par les médias, a aussi été étudié comme un phénomène à part entière (Westphal et Deephouse, 2011).

En termes de conséquences concrètes, les parties prenantes ont tendance à éviter les organisations faisant face à la désapprobation ou au stigmate (Devers, et al 2009). Les entreprises cèdent leurs actifs dans les industries stigmatisées (Durand et Vergne, 2015), abandonnent leurs alliés stigmatisés (Bruyaka et al., A paraître) et s'engagent dans un certain nombre de pratiques défensives (Canberry et King, 2012) notamment pour gérer leur image (McDonnell et King, 2013). Les employés des organisations désapprouvées sont généralement moins satisfaits au travail, à moins qu'ils ne considèrent les critiques extérieures comme injustifiées, auquel cas la désapprobation de leur organisation peut avoir un effet positif sur leur satisfaction au travail (Roulet, 2017). Enfin, le stigmate généré par l'échec perturbe l'apprentissage qui peut en résulter (Dahlin, Chuang et Roulet, 2018). Le stigmate peut cependant se transmettre des organisations aux individus qui interagissent avec elles (Hudson et Okhuysen, 2009), d'où la nécessité de prendre en compte plusieurs niveaux d'analyse dans l'étude des évaluations sociales.

\subsection{Le niveau d'analyse : au-delà et en-deca de l'organisation}

Comme nous l'avons noté, la notion de stigmate a pris racine dans les travaux sur le stigmate au niveau individuel initié par Goffman (1963). La littérature sur le stigmate est aussi liée intimement au concept de «dirty work» qui désigne les occupations méprisées (Ashforth et Kreiner, 1999). La façon dont les différents niveaux d'analyse s'informent les uns les autres est cruciale pour comprendre les antécédents et conséquences des évaluations sociales. En effet, la stigmatisation des minorités au sein des organisations peut porter l'opprobre sur ces organisations (Stenger et Roulet, 2017) en fonction de la façon dont ce stigmate est géré par l'individu et son organisation (Jones et King, 2014). La stigmatisation des entrepreneurs peut 
aussi mener à l'échec organisationnel (Cusin et Maymo, 2017). Inversement, les organisations stigmatisées peuvent communiquer à leurs membres un stigmate individuel comme le montre les travaux sur les saunas gay qui affectent la vie sociale de leurs usagers (Hudson et Okhuysen, 2009) ou les entreprises en faillite qui pénalisent leurs anciens employés (Sutton et Callahan, 1987).

En montant d'un niveau d'analyse et en passant à un échelon plus macro, on trouve de nombreux travaux sur les industries stigmatisées (Durand et Vergne, 2015) ou contestées (Galvin, Ventresca et Hudson, 2004). Les industries stigmatisées sont généralement associées à des professions stigmatisées (Voss, 2015 ; Roulet, 2015a). Généralement les membres de ces industries ou champs, cherchent à éviter l'association avec cette industrie qui forme une catégorie stigmatisée (Durand et Vergne, 2015). Comme le soulignent Mishina et Devers (2012), le processus de stigmatisation tend à regrouper les organisations stigmatisées par groupes subissant ce label négatif.

En ce qui concerne les évaluations sociales positives, les recherches au niveau individuel peuvent documenter la perception des organisations, comme par exemple dans le cas du leadership (Blom et Alvesson, 2015). La légitimité, parce qu'elle implique le respect de normes sociétales ou organisationnelles (soit à un niveau macro), est, par nature, un concept qui se nourrit d'analyse multi-niveaux (Suddaby et al., 2017). Le statut peut faire l'objet d'enjeu de pouvoir et donc de conflit dans les équipes (Piazza et Castellucci, 2015). La littérature sur le statut fait souvent le lien entre statut des organisations et statut des individus, membres de ces organisations (Ertug et Castellucci, 2013 ; Piazza et Castellucci, 2015), mais l'étude de tels phénomènes nécessite d'observer les mouvements plutôt que le stock d'individus pour contourner les problèmes d'endogénéité.

Le diagramme 1 résume le double continua des évaluations sociales positives et négatives et présente les différentes superpositions des concepts. Comme nous l'avons souligné, la légitimité pragmatique peut jouer un rôle réputationnel tout comme le rang dans des classements peut signaler à la fois le statut et la réputation (Barnett, et al. 2006). L'évaluation par les pairs est fonction de l'adhérence aux normes mais aussi du statut (Shymko et Roulet, 2017). Du cote des évaluations sociales négatives, la désapprobation peut être associée à la variance au sein d'une catégorie stigmatisée (Piazza et Perretti 2015).

Diagramme 1 : Les continua des évaluations sociales positives et négatives 


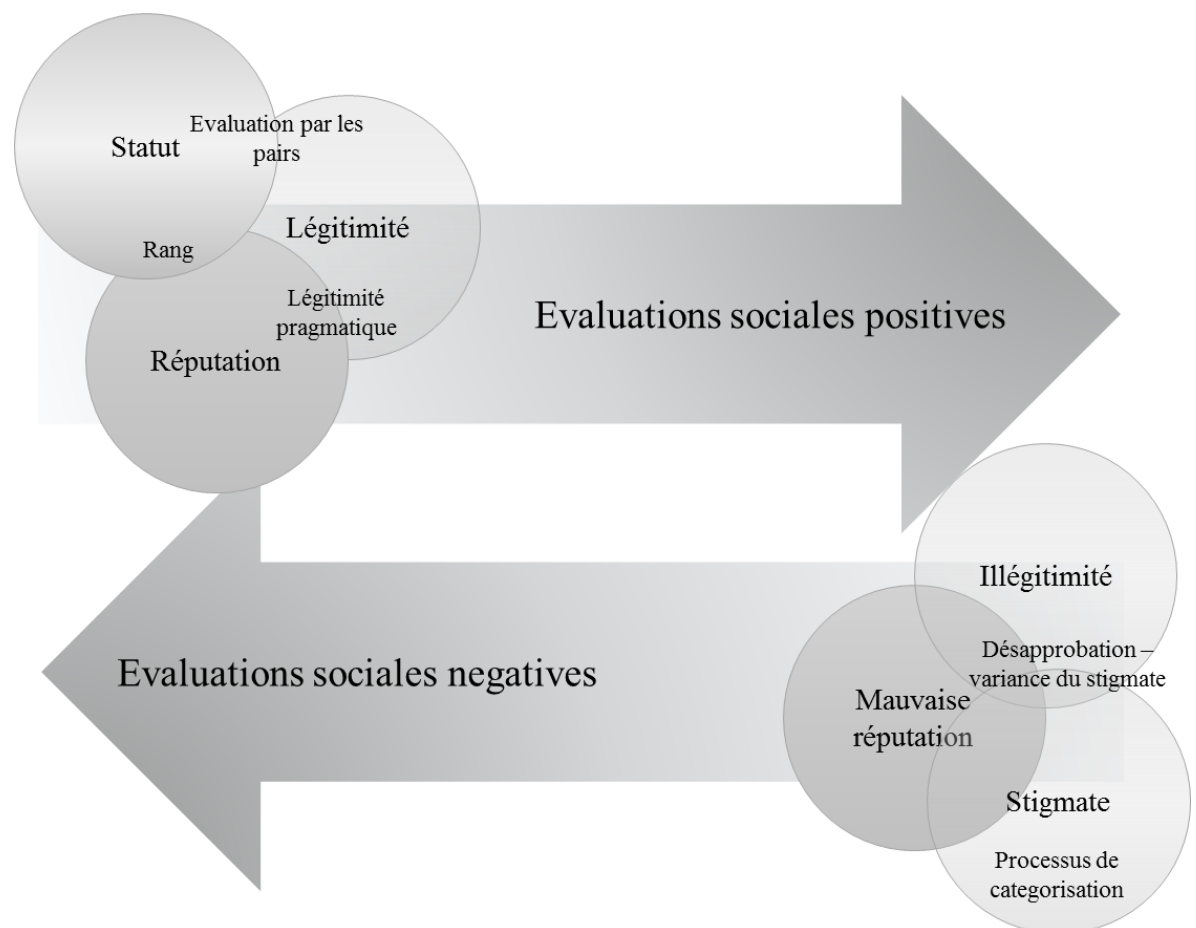

\section{MECHANISMES, APPROCHES THEORIQUES ET EMPIRIQUES}

Dans cette deuxième section, nous explorons les mécanismes théoriques sur lesquels sont construits la compréhension des antécédents et conséquences des évaluations sociales en stratégie et théorie des organisations. En effet, les évaluations sociales ne sont pas des théories en elles-mêmes mais plutôt des concepts qui peuvent servir à informer d'autres théories ou peuvent se construire et s'étoffer sur la base d'autres cadres conceptuels. Nous distinguons ici les différentes approches théoriques liées aux évaluations sociales et le rôle crucial que jouent les médias et les audiences, dans la théorisation des évaluations sociales.

\subsection{Approche sociologique ou stratégique ?}

Suchman (1995) distingue une vision stratégique ou institutionnaliste de la légitimité. La théorie néo-institutionnelle a en effet de longue date permis de comprendre les dynamiques de la légitimité (Suddaby, Bitektine et Haack, 2017) mais aussi d'autres évaluations sociales comme le stigmate (Roulet, 2015a). La légitimité traduisant la conformité aux normes et donc la proximité aux institutions, on peut percevoir ce concept comme directement ancré dans une perspective institutionnaliste (Roulet, 2015b). Mais tous les travaux ne font pas forcément le choix de s'ancrer dans cette perspective. La distinction de Suchman (1995) est motivée par le fait que l'approche institutionnaliste minimise le rôle de 
l'organisation dans sa faculté à acquérir ou agir sur sa propre légitimité et celle d'autres acteurs. A contrario, la perspective stratégique reconnait le pouvoir des organisations à acquérir de la légitimité en manipulant leur conformité aux normes mais aussi en changeant les normes elles-mêmes (Bitektine, 2011). En somme, la perspective stratégique reconnaît les évaluations sociales comme des actifs intangibles de l'organisation (voir par exemple Gaultier-Gaillard et Pratlong, 2011), et c'est pourquoi cette dernière se focalise généralement sur le rôle de la réputation qui paraît plus manipulable (Deephouse et Carter, 2005). Cette approche stratégique s'est naturellement construite sur la théorie des ressources (Deephouse, 2000 ; Barnett, et al., 2006) et fait l'hypothèse que ces évaluations sociales positives sont plus utiles dans certains marchés qui reposent sur les signaux intangibles de qualité (Shymko et Roulet, 2017).

\subsection{Catégorisation, identité et audiences}

La théorie de la catégorisation a récemment émergé comme une approche cruciale à la compréhension des conséquences et de la formation des évaluations sociales (voir la revue de littérature de Vergne et Wry, 2014). En particulier, la stigmatisation a été conceptualisée comme un processus de catégorisation (l'organisation fait partie de la catégorie stigmatisée, ou est à l'extérieur de cette catégorie) (voir Vergne, 2012). La catégorisation est définie comme une classification cognitive partagée par les parties prenantes (Durand et Paolella, 2013). D'autres travaux récents utilisent l'outil conceptuel des catégories pour comprendre les évaluations sociales : ainsi le «category spanning » (le fait d'appartenir à plusieurs catégories plutôt qu'une seule) est traditionnellement considéré comme ayant des conséquences néfastes sur la manière dont est évaluée l'organisation et au final sa performance. Paolella et Durand (2016) trouvent des résultats inverses en théorisant la perspective des audiences et la façon dont ces audiences valorisent certaines caractéristiques de l'organisation. La catégorisation est aussi devenue progressivement le pendant évaluatif de l'écologie des organisations qui vise à expliquer la survie et la disparition des organisations (Vergne et Wry, 2014). Enfin, l'appartenance aux catégories est un élément potentiellement flexible qui peut être manipulé pour améliorer l'évaluation sociale de l'entreprise comme le montre Vergne (2012) dans son étude de l'industrie de l'armement.

Ainsi, la théorie des catégories valorise le rôle des audiences pour comprendre l'influence des évaluations sociales, comme le font d'autres travaux récents qui théorisent les liens entre les évaluations sociales formulées par différentes parties prenantes (Boutinot et al., 
2017 ; Shymko et Roulet, 2017). En effet, les audiences affectent la vie économique et sociale de l'organisation de façon parfois variée. Certaines audiences peuvent formuler une évaluation sociale négative sans que cela affecte la performance ou plus généralement la destinée d'une organisation. A contrario, les évaluations formulées les clients, les employés, les organes de régulations dans certaines industries, peuvent avoir un effet de vie ou de mort sur les organisations. Ces évaluations sont elles-mêmes directement liées et influencées par les évaluations formulées par d'autres audiences ou parties prenantes telles que les médias ou le grand public (Clemente et Roulet, 2015 ; Roulet et Clemente, A paraître).

La théorie de l'identité sociale, qui peut aussi révéler l'adhérence à des catégories, est une autre approche permettant une meilleure compréhension des évaluations sociales. Le stigmate des organisations génère un travail identitaire de la part de ces membres (Hudson et Okhuysen, 2009 ; Tracey et Phillips, 2016 ; Hampel et Tracey, 2016) de par l'impact négatif que celui-ci peut avoir sur l'estime de soi pour ces derniers (Roulet, 2017). L'appartenance à une catégorie stigmatisée (par exemple une occupation ternie et méprisée) interagit avec l'identité des individus et des organisations, renforçant même parfois le sentiment d'appartenance comme réaction à l'opprobre publique (Ashforth et Kreiner, 1999).

\subsection{Scandale et dynamiques de diffusion}

Les processus de formation et transmission des évaluations sociales n'est que partiellement documenté. Certains travaux notables examinent le rôle des scandales - le processus à travers lequel une évaluation négative émerge puis se diffuse (Adut, 2008 ; Kuhn et Ashcraft, 2003 ; Clemente et Gabbioneta, 2017). Roulet et Clemente (2017) expliquent le concept de cascade informationnelle, ou lorsque les évaluations sociales sont transmises d'un medium ou d'une audience à une autre. La cause des évaluations sociales négatives épisodiques (par exemple, le stigmate ponctuel plutôt que stigmate par nature d'après Hudson, 2008) la plus généralement admise est celle des comportements illicites, amoraux ou illégaux (Vaughan, 1999 ; Muzio et al., 2016). Ces comportements sont alors rendus publics par les médias qui eux-mêmes peuvent représenter une diversité de perspective sur ces comportements (Clemente et Gabbioneta, 2017). Les écarts de conduite ne sont pas pour autant forcément sources de scandale, s'ils ne sont pas identifiés, en particulier en l'absence de media qui peuvent jouer le rôle d'une caisse de résonnance (Palmer, 2012 ; Etter, et al. 2018 ; Daudigeos, Roulet et Valiorgue, A paraître). 
En somme, certains comportements répréhensibles peuvent rester ignorés, ne pas être repris par les médias, et donc ne pas faire l'objet d'un effet sur les évaluations sociales. Les médias n'attribuent pas forcément leur attention de manière rationnelle ou proportionnelle à la gravité des évènements identifiés (Petkova et al. 2013 ; Roulet et Clemente, 2017). Les médias sont sujets à un certain nombre de biais sur la façon dont ils transmettent les évaluations sociales et les causes de ces évaluations (Roulet, 2015b). L'importance des réseaux organisationnels est cruciale pour comprendre les dynamiques du statut (Washington et Zajac, 2005) mais aussi la marginalisation ou l'intégration des acteurs stigmatisés (Delacour et Liarte, 2013).

Les évaluations sociales sont, par nature, dynamiques. La réputation peut, par exemple, être « réparée » (Rhee et Valdez, 2009). La légitimité peut s'éroder dans le temps (Suddaby et al., 2017) notamment car les institutions évoluent, ce qui affecte le degré de conformité aux normes des organisations (Roulet, 2015a ; Clemente, Durand et Roulet, 2017). Le statut évolue avec le capital humain des organisations (Piazza et Castellucci, 2015) et est particulièrement dépendant des conditions de liquidité et de la qualité du marché du travail, mais est considéré comme moins friable que la réputation (Ertug et Castellucci, 2013). Légitimité, réputation et statut sont intimement et récursivement liés : par exemple, une entreprise peut bénéficier d'un statut élevé, lui permettant sur le long terme de construire une meilleure réputation en offrant des services ou des produits de plus grande qualité. Ces nouvelles ressources peuvent ensuite permettre à l'entreprise d'investir dans une meilleure conformité aux normes de responsabilité sociale en vue d'acquérir plus de légitimité (Reynaud et Walas, 2015 ; Bothello et Djelic, 2017).

\subsection{Défis méthodologiques}

Les travaux sur les évaluations sociales donnent lieu à la fois à des recherches qualitatives (par exemple, Hudson et Okhuysen, 2009 ; Reynaud et Walas, 2015 ; Tracey et Phillips, 2016) mais aussi quantitatifs (par exemple, Vergne, 2012 ; Phillippe et Durand, 2011 ; Roulet, 2015b) et mixtes (par exemple, Roulet, 2015a ; Shymko et Roulet, 2017).

Comme le notent Hudson et Okhuysen (2009) dans leurs travaux sur le stigmate des saunas gay ou Wolfe et Blithe (2015) dans leurs travaux sur les maisons closes, étudier des industries stigmatisées peut revêtir de nombreux challenges méthodologiques. Pour les chercheurs qualitatifs, comment avoir accès au champ étudié ? Comment ne pas perturber 
l'observation par sa présence de chercheur? L'observation participante à couvert ou partiellement à couvert est particulièrement courant pour ce type de situation (Roulet, et al., 2017). Dans le contexte d'industries contestées, il est en effet plus difficile pour les chercheurs de collecter des données, de par la discrétion des organisations examinées. Pour les travaux quantitatifs, les données au niveau organisationnel sont rares et il est courant de prendre un niveau d'analyse plus macro (voir par exemple le travail de Piazza et Jourdan, A paraître sur l'effet des scandales sur la compétition dans le champ du religieux) et de rechercher des données longitudinales pour pallier au faible nombre d'individus au sein des milieux ou industries contestés. Une autre approche qui permet de résoudre le problème du faible nombre d'acteurs dans les champs stigmatisées (et donc le petit nombre de cas ou d'observations) est notamment l'analyse qualitative comparée (voir Vergne et Depeyre, 2016).

Sur le plan quantitatif, le traitement de contenu média est l'une des méthodes dominantes pour capturer le stigmate, la réputation, la légitimité ou l'illégitimité (voir par exemple Etter et al, 2018 ; Westphal et Deephouse, 2011 ; Vergne, 2012 ; Roulet, 2015b). Ces méthodes se fondent sur la transformation en variables quantitatives d'une base d'articles de journaux dépeignant une organisation. L'unité d'analyse peut être l'article de presse (combien d'articles de presse sont-ils négatifs ?) comme dans l'étude de Vergne (2012)). Mais ces méthodes peuvent aussi évaluer la négativité du vocabulaire au sein de ces articles (Roulet, 2015b).

Enfin, les approches historiques sont particulièrement utiles pour explorer les dynamiques des évaluations sociales. Comment les organisations deviennent-elles légitimes ? A quel horizon temporel ? On peut, par exemple, citer le travail historique sur la légitimation de l'absinthe (Ben Slimane et al., 2016). Le statut est une évaluation plus persistante et inerte que la réputation (Ertug et Castellucci, 2013) mais cette comparaison doit encore être approfondie. Les chercheurs peuvent reconstruire de manière narrative l'histoire des industries (Gill et al., 2017) et établir la variation historique dans les processus de légitimation et de contestation sur des périodes longues. L’industrie financière, par exemple, a traversé de nombreux cycles de légitimation et délégitimation (Roulet, 2015a ; 2015b).

\section{AGENDA DE RECHERCHE ET PERSPECTIVES}


Nous avons déjà identifié un certain nombre de questions de recherche qui restent à explorer dans le champ des évaluations sociales. Par exemple, nous avons évoqué les dynamiques temporelles et historiques des évaluations sociales, mais aussi le développement de méthodes adéquates pour palier au faible nombres d'acteurs dans les champs stigmatisés et l'accès au terrain dans les milieux contestés. Dans cette section, nous nous focalisons sur deux évolutions récentes et plus générales des travaux sur les évaluations sociales en stratégie, qui sont aussi deux points qui nécessitent de nouveaux éclaircissements et perspectives.

\subsection{Convergence et divergence des évaluations sociales}

Comme nous l'avons souligné, la définition des différentes évaluations sociales continue de poser problème de par la façon dont les concepts sont utilisés parfois de manière ambiguë (voir par exemple l'usage du concept de réputation, à la frontière de la légitimité dans McDonnell et King, 2013 ; ou Zavyalova et al., 2016). Ces concepts ne sont généralement pas interchangeables, et l'opérationnalisation de ces évaluations révèlent généralement plus de problèmes qu'il n'en résout. Certains travaux ont cependant fait l'effort notable de bien différencier ces concepts et même de les examiner dans le même contexte (voir notamment Philippe, 2006 ; 2009 ; Philippe et Durand, 2011). Parmi ces travaux, Deephouse et Carter (2005) observent le lien récursif entre performance d'un côté et légitimité et réputation de l'autre ; Ertug et Castellucci (2013) étudient, eux, l'effet conjoint de la réputation et du statut.

Comme nous l'avons souligné, clarifier le rôle des médias et des audiences devraient permettre de mieux différencier ces différents concepts. La légitimité est généralement une évaluation formée par des parties prenantes extérieures au champ d'action de l'organisation (par exemple la société dans son ensemble, les ONG, les gouvernements) tandis que la réputation provient des parties prenantes influençant directement la performance et la survie de l'organisation (par exemple les clients ou les régulateurs). Les audiences n'agissent pas indépendamment les unes des autres et il existe des liens entre les différentes évaluations qu'elles formulent (Roulet, 2015b; Shymko et Roulet, 2017). Il existe des signaux entre audiences - par exemple, une attaque des médias sur une entreprise affecte la façon dont les employés de cette entreprise perçoivent leur employeur (Roulet, 2017). Les audiences jouent un rôle essentiel pour comprendre l'impact et les antécédents des évaluations sociales. Plus les audiences ont un pouvoir sur l'organisation visée, plus l'évaluation qu'elles formulent ont une importance cruciale pour la performance et la survie de cette organisation. 


\subsection{L'évaluation sociale comme variable dépendante}

Comme expliqué en introduction, de nombreux travaux examinent désormais les évaluations sociales comme une variable intéressante en elle-même et pas seulement un déterminant d'autres variables comme la performance. Le rôle des évaluations sociales sur la performance est établi empiriquement (Deephouse et Carter, 2005) mais est de plus en plus souvent perçu comme un médiateur (Paolella et Durand, 2016) affecté par le comportement des organisations. Par exemple, de nombreux travaux examinent le rôle de la responsabilité sociale et communication environnementale pour rendre les entreprises légitimes ou réputées (Philippe, 2006 ; Philippe et Durand, 2011 ; Reynaud et Walas, 2015 ; Bothello et Djelic, 2017). De nombreux travaux étudient aussi la façon dont les entreprises peuvent gérer leur mauvaise image (Westphal et Deephouse, 2011 ; Vergne, 2012 ; McDonnell et King, 2013). Ces travaux sur les évaluations sociales comme variable d'intérêt en elle-même rejoignent la perspective stratégique des évaluations sociales (Suchman, 1995) dans le sens où elles perçoivent ces évaluations comme des actifs intangibles (Deephouse, 2000 ; Barnett, et al., 2006).

Certains secteurs comme le conseil ou l'audit (les «professional services firms ») reposent tout particulièrement sur des consommation et production effrénées d'évaluation sociale (Stenger et Roulet, 2017). Les évaluations sociales elles-mêmes peuvent être plus ou moins légitime pour les organisations : les banques d'investissement portent peu de crédit à la façon dont elles sont perçues par les médias par rapport à la façon dont elles sont perçues par leurs pairs (Roulet, 2015a; 2015b) tandis que par contraste la reconnaissance des organisations par leurs pairs peut n'avoir aucune valeur pour des parties prenantes extérieures au champ (Shymko et Roulet, 2017). Les chercheurs en management sont eux aussi soumis à toute sorte d'évaluations sociales (Mangematin et Belkhouja, 2015). Ces évaluations sont constantes et cruciales pour la carrière des chercheurs tant elles sont utilisées par les comités de promotion ou de titularisation. Le champ académique est même un milieu qu'on pourrait définir comme lui aussi extrêmement dépendant aux évaluations sociales et dans ce sens, son étude pourrait être particulièrement adaptée pour comprendre les antécédents et conséquences des évaluations sociales. Comment la visibilité des chercheurs affecte-t-elle leur nombre de citations ? Comment les chercheurs identifiés pour leur mauvaise conduite (par exemple, la falsification des données) sont-ils stigmatisés et isolés du reste du champ ? Cette «ultradépendance » a évidemment de nombreux effets néfastes qu'ils conviendraient d'étudier. Les 
évaluations sociales peuvent être la conséquence de biais liés au genre, l'ethnicité ou la sexualité, ce qui compromet la notion d'équité dans ces champs.

\section{CONCLUSION}

Les évaluations sociales et leurs nombreuses conceptualisations fournissent une boite à outil riche et complexe pour comprendre comment les perceptions collectives affectent la vie économique des organisations. Dans cette revue de littérature, nous mettons en exergue les divergences et convergences de définitions, mais aussi la grande richesse théorique que ces concepts ont à offrir. Légitimité, réputation et statut ont une très grande variété de dimensions (voir par exemple les suggestions de Bitektine, 2011 ou Boutinot et al., 2017), ce qui amène les chercheurs à les confondre régulièrement. Mais ces dimensions permettent aussi de distinguer de manière fine la façon dont ces évaluations sociales peuvent servir de ressources, être acquises, et perdues.

Le développement d'une littérature distincte sur les évaluations sociales négatives permet d'enrichir encore plus ce panorama pour mieux prendre en considération les phénomènes récents de scandales financiers ou les transgressions éthiques par les entreprises de Enron à Volkswagen (Clemente et Gabbioneta, 2017). Ces cas ne montrent pas seulement que la légitimité, la réputation ou le statut peuvent être perdus à la suite d'écarts de conduite mais que l'image de cette organisation peut aussi être entachée, la transportant ainsi sur un continuum d'évaluations sociales négatives. Les entreprises peuvent-elles survivre sans légitimité, réputation ou statut? Le cas échéant peuvent-elles survivre en étant stigmatisées ? Bien que les travaux existants répondent par la positive à ces questions (Hudson, 2008 ; Hudson et Okhuysen, 2009), il reste à explorer pleinement le «comment » de ce phénomène. Un développement de méthodes adaptées, la conceptualisation du rôle des audiences et des médias, et les approches longitudinales et historiques devraient permettre d'approfondir notre compréhension des évaluations sociales et de leurs conséquences. 


\section{REFERENCES}

Adut, A. (2008). On scandal: Moral disturbances in society, politics, and art. Cambridge: Cambridge University Press.

Anicich, E. M., Fast, N. J., Halevy, N., \& Galinsky, A. D. (2015). When the bases of social hierarchy collide: Power without status drives interpersonal conflict. Organization Science, 27(1), 123-140.

Ashforth, B. E., \& Kreiner, G. E. (1999). "How can you do it?": Dirty work and the challenge of constructing a positive identity. Academy of Management Review, 24(3), 413434.

Barnett, M. L., Jermier, J. M., \& Lafferty, B. A. (2006). Corporate reputation: The definitional landscape. Corporate reputation review, 9(1), 26-38.

Benjamin, B. A., \& Podolny, J. M. (1999). Status, quality, and social order in the California wine industry. Administrative science quarterly, 44(3), 563-589.

Ben Slimane, K. Chaney, D., Vaara, E. et Wang, T. (2016) Between memories and market. Relegitimation of absinthe in France since 1980s. EGOS Proceedings 2016.

Bitektine, A. (2011). Toward a theory of social judgments of organizations: The case of legitimacy, reputation, and status. Academy of Management Review, 36(1), 151-179.

Blom, M., \& Alvesson, M. (2015). Less followership, less leadership? An inquiry into the basic but seemingly forgotten downsides of leadership.M@n@gement, 18(3), 266-282.

Bothello, J., \& Salles-Djelic, M. L. (2017). Evolving Conceptualizations of Organizational Environmentalism: A Path Generation Account. Organization Studies, 0170840617693272.

Boutinot, A., Joly, I., Mangematin, V., \& Ansari, S. (2017). Exploring the links between reputation and fame: Evidence from French contemporary architecture. Organization Studies, 0170840616670433.

Bruyaka, O., Philippe, D. et Castaner, X. (A paraître). Run Away or Stick Together? The Impact of Organization-Specific Adverse Events on Alliance Partner Defection. Academy of Management Review.

Carberry, E. J., \& King, B. G. (2012). Defensive practice adoption in the face of organizational stigma: Impression management and the diffusion of stock option expensing. Journal of Management Studies, 49(7), 1137-1167. 
Carter, R., \& Manaster, S. (1990). Initial public offerings and underwriter reputation. the Journal of Finance, 45(4), 1045-1067.

Castellucci, F., \& Ertug, G. (2010). What's in it for them? Advantages of higher-status partners in exchange relationships. Academy of Management Journal, 53(1), 149-166.

Chiambaretto, P. (2015). Resource dependence and power-balancing operations in alliances: The role of market redefinition strategies.M@n@gement, 18(3),205-233.

Clemente, M., \& Gabbioneta, C. (2017). How does the media frame corporate scandals? The case of German newspapers and the Volkswagen diesel scandal. Journal of Management Inquiry, 1056492616689304.

Clemente, M., \& Roulet, T. J. (2015). Public opinion as a source of deinstitutionalization: A “spiral of silence” approach. Academy of Management Review, 40(1), 96-114.

Clemente, M., Durand, R., \& Roulet, T. (2017). The recursive nature of institutional change: An Annales School perspective. Journal of Management Inquiry, 26(1), 17-31.

Collet, F., \& Philippe, D. (2014). From hot cakes to cold feet: A contingent perspective on the relationship between market uncertainty and status homophily in the formation of alliances. Journal of Management Studies, 51(3), 406-432.

Cusin, J., \& Maymo, V. (2016). Post-bankruptcy stigmatization of entrepreneurs and bankers'decisions to finance.M@n@gement, 19(4), 305.

Dahlin, K., Chuang, Y. T., \& Roulet, T. (2018). Opportunity, motivation and ability to learn from failures and errors: Review, synthesis, and the way forward. Academy of Management Annals, 12(1), 252-277.

Daudigeos, T., Roulet, T., \& Valiorgue, B. (A paraître). How Scandals Act as Catalysts of Fringe Stakeholders' Contentious Actions Against Multinational Corporations. Business \& Society.

Delacour, H., \& Liarte, S. (2013). «Je t'aime... Moi non plus!»: De la marginalisation à l'intégration de l'industrie du cinéma pornographique dans le réseau BluRay.M@n@gement,16(3),238-263.

Deephouse, D.L., (2000). Media reputation as a strategic resource: An integration of mass communication and resource-based theories. Journal of management, 26(6), pp.1091- 1112.

Deephouse, D. L., \& Carter, S. M. (2005). An examination of differences between organizational legitimacy and organizational reputation. Journal of management Studies, 42(2), 329-360. 
Deephouse, D. L., \& Suchman, M. (2008). Legitimacy in organizational institutionalism. The Sage handbook of organizational institutionalism, 49, 77.

Devers, C. E., Dewett, T., Mishina Y. and Belsito C. A. (2009). 'A general theory of organizational stigma'. Organization Science, 20(1): 154-171

Dowling, J., \& Pfeffer, J. (1975). Organizational legitimacy: Social values and organizational behavior. Pacific sociological review, 18(1), 122-136.

Durand, R., \& Paolella, L. (2013). Category stretching: Reorienting research on categories in strategy, entrepreneurship, and organization theory. Journal of Management Studies, 50(6), $1100-1123$.

Durand, R., \& Vergne, J. P. (2015). Asset divestment as a response to media attacks in stigmatized industries. Strategic Management Journal, 36(8), 1205-1223.

Dutton, J. E., \& Dukerich, J. M. (1991). Keeping an eye on the mirror: Image and identity in organizational adaptation. Academy of management journal, 34(3), 517-554.

Elsbach, K. D., \& Kramer, R. M. (1996). Members' responses to organizational identity threats: Encountering and countering the Business Week rankings. Administrative Science Quarterly, 41(3): 442-476.

Elsbach, K. D., \& Sutton, R. I. (1992). Acquiring organizational legitimacy through illegitimate actions: A marriage of institutional and impression management theories. Academy of management Journal, 35(4), 699-738.

Ertug, G., \& Castellucci, F. (2013). Getting what you need: How reputation and status affect team performance, hiring, and salaries in the NBA. Academy of Management Journal, 56(2), 407-431.

Etter, M., Colleoni, E., Illia, L., Meggiorin, K., \& D’Eugenio, A. (2018). Measuring organizational legitimacy in social media: Assessing citizens' judgments with sentiment analysis. Business \& Society, 57(1), 60-97.

Fombrun, C., \& Shanley, M. (1990). What's in a name? Reputation building and corporate strategy. Academy of management Journal, 33(2), 233-258.

Galvin, T. L., Ventresca, M. J., \& Hudson, B. A. (2004). Contested industry dynamics. International Studies of Management \& Organization, 34(4), 56-82.

Gaultier-Gaillard, S., \& Pratlong, F. (2011). Le risque de réputation: le cas du secteur bancaire. Management \& Avenir, (8), 272-288. 
Gill, M. J., Gill, D. J., \& Roulet, T. J. (2018). Constructing Trustworthy Historical Narratives: Criteria, Principles and Techniques. British Journal of Management, 29(1), 191205.

Glass, G. V., \& Ellett Jr, F. S. (1980). Evaluation research. Annual review of psychology, 31(1), 211-228.

Goffman, E. (1963). Stigma: Notes on the Management of Spoiled Identity. Prentice-Hall, Englewood Cliffs, NJ.

Hampel, C. E., \& Tracey, P. (2017). How organizations move from stigma to legitimacy: The case of cook's travel agency in Victorian Britain. Academy of Management Journal, 60(6), 2175-2207.

Helms, W. S., \& Patterson, K. D. (2014). Eliciting Acceptance For "Illicit" Organizations: The Positive Implications of Stigma for MMA Organizations. Academy of Management Journal, 57(5), 1453-1484.

Hudson, B. A. (2008). Against all odds: A consideration of core-stigmatized organizations. Academy of Management Review, 33(1), 252-266.

Hudson, B. A., \& Okhuysen, G. A. (2009). Not with a ten-foot pole: Core stigma, stigma transfer, and improbable persistence of men's bathhouses. Organization Science, 20(1), 134153.

Jones KP and King EB (2014) Managing concealable stigmas at work: a review and multilevel model. Journal of Management 40(5): 1466-94.

Kuhn, T., \& Ashcraft, K. L. (2003). Corporate Scandal and the Theory of the Firm Formulating the Contributions of Organizational Communication Studies. Management Communication Quarterly, 17(1), 20-57.

Lamont, M. (2012). Toward a comparative sociology of valuation and evaluation. Annual Review of Sociology, 38:201-21 .

Link B.G and Phelan J. C. (2001). Conceptualizing Stigma. Annual Review of Sociology, 27, pp. 363-395.

Mangematin, V., \& Belkhouja, M. (2015). A Note On The Evolution Of The French Management Scholarship,1994-2014.M@n@gement, 18(3), 194.

McDonnell, M. H., \& King, B. (2013). Keeping up appearances reputational threat and impression management after social movement boycotts. Administrative Science Quarterly, 58(3), 387-419. 
Mishina, Y., \& Devers, C. E. (2012). On being bad: Why stigma is not the same as a bad reputation. The Oxford handbook of corporate reputation, 201-220.

Muzio D, Faulconbridge J, Gabbioneta C and Greenwood R (2016) Bad Apples, Bad Barrels and Bad Cellars: A 'Boundaries' Perspective on Professional Misconduct. In Palmer D, Greenwood R and Smith-Crowe K (eds) Organizational Wrongdoing. Cambridge, UK: Cambridge University Press: 141-175.

Palmer, D. (2012). Normal organizational wrongdoing: A critical analysis of theories of misconduct in and by organizations. Oxford University Press: Oxford.

Palmer D, Greenwood R and Smith-Crowe K (eds) (2016) Organizational Wrongdoing. Cambridge, UK: Cambridge University Press.

Paolella, L., \& Durand, R. (2016). Category spanning, evaluation, and performance: Revised theory and test on the corporate law market. Academy of Management Journal, 59(1), 330-351.

Petkova, A. P., Rindova, V. P., \& Gupta, A. K. (2013). No news is bad news: Sensegiving activities, media attention, and venture capital funding of new technology organizations. Organization Science, 24(3), 865-888.

Piazza, A., \& Castellucci, F. (2014). Status in organization and management theory. Journal of Management, 40(1), 287-315.

Piazza, A., \& Jourdan, J. (A paraître). When the Dust Settles: The Consequences of Scandals for Organizational Competition. Academy of Management Journal.

Piazza, A., \& Perretti, F. (2015). Categorical stigma and firm disengagement: Nuclear power generation in the United States, 1970-2000. Organization Science, 26(3), 724-742.

Philippe, D. (2006). La communication environnementale organisationnelle comme stratégie de légitimation. Management international, 10(3), 49.

Philippe, D. (2009). Social perceptions and the structuration of markets: Three essays on the impacts of conforming and framing strategies on organizational status, reputation and legitimacy (Doctoral dissertation, Jouy-en-Josas, HEC).

Philippe, D., \& Durand, R. (2011). The impact of norm-conforming behaviors on firm reputation. Strategic Management Journal, 32(9), 969-993.

Podolny, J. M. (1993). A status-based model of market competition. American journal of sociology, 98(4), 829-872. 
Podolny, J. M. (2010). Status signals: A sociological study of market competition. Princeton University Press.Rhee, M., \& Valdez, M. E. (2009). Contextual factors surrounding reputation damage with potential implications for reputation repair. Academy of Management Review, 34(1), 146-168.

Reynaud, E., \& Walas, A. (2015). Discours sur la RSE dans le processus de légitimation de la banque. Revue française de gestion, (3), 187-209.

Roberts, P. W., \& Dowling, G. R. (2002). Corporate reputation and sustained superior financial performance. Strategic management journal, 23(12), 1077-1093.

Roulet, T. J. (2015a). "What good is Wall Street?" Institutional contradiction and the diffusion of the stigma over the finance industry. Journal of Business Ethics, 1-14.

Roulet, T. (2015b). Qu'il est bon d'être méchant ! Paradoxe de l'illégitimité organisationnelle dans le contexte des banques d'investissement. Revue française de gestion, $41(248), 41-55$.

Roulet, T. J. (2017). Good to be disliked? Exploring the relationship between disapproval of organizations and job satisfaction in the French context. Journal of General Management, 42(4), 68-79.

Roulet T. \& Clemente, M. (A paraître) Let's Open Media's Black Box: Media as a Set of Heterogeneous Actors and not only as a Homogenous Ensemble. Academy of Management Review

Roulet, T. J., Gill, M. J., Stenger, S., \& Gill, D. J. (2017). Reconsidering the value of covert research: the role of ambiguous consent in participant observation. Organizational Research Methods, 1094428117698745.

Shymko, Y., \& Roulet, T. J. (2017). When does Medici hurt da Vinci? Mitigating the signaling effect of extraneous stakeholder relationships in the field of cultural production. Academy of Management Journal, 60(4), 1307-1338.

Stenger, S. \& Roulet, T. (A paraître) Pride against Prejudice? The Stakes of Concealment and Disclosure of a Stigmatized Identity for Gay and Lesbian Auditors. Work, Employment \& Society

Suchman, M. C. (1995). Managing legitimacy: Strategic and institutional approaches. Academy of management review, 20(3), 571-610.

Suddaby, R., Bitektine, A., \& Haack, P. (2017). Legitimacy. Academy of Management Annals, 11(1), 451-478. 
Sutton, R. I., \& Callahan, A. L. (1987). The stigma of bankruptcy: Spoiled organizational image and its management. Academy of Management journal, 30(3), 405-436.

Tracey, P., \& Phillips, N. (2016). Managing the consequences of organizational stigmatization: Identity work in a social enterprise. Academy of Management Journal, 59(3), 740-765.

Turban, D. B., \& Greening, D. W. (1997). Corporate social performance and organizational attractiveness to prospective employees. Academy of management journal, 40(3), 658-672.

Vaughan, D. (1999). The dark side of organizations: Mistake, misconduct, and disaster. Annual review of sociology, 25(1), 271-305.

Vergne, J. P. (2012). Stigmatized categories and public disapproval of organizations: A mixed-methods study of the global arms industry, 1996-2007. Academy of Management Journal, 55(5), 1027-1052.

Vergne, J. P., \& Depeyre, C. (2016). How do firms adapt? A fuzzy-set analysis of the role of cognition and capabilities in US defense firms' responses to 9/11. Academy of Management Journal, 59(5), 1653-1680.

Vergne, J. P., \& Wry, T. (2014). Categorizing categorization research: Review, integration, and future directions. Journal of Management Studies, 51(1), 56-94.

Voss, G. (2015). Stigma and the Shaping of the Pornography Industry. Routledge.

Wang, T., Wezel, F. C., \& Forgues, B. (2016). Protecting market identity: When and how do organizations respond to consumers' devaluations?. Academy of Management Journal, 59(1), 135-162.

Walker, K. (2010). A systematic review of the corporate reputation literature: Definition, measurement, and theory. Corporate reputation review, 12(4), 357-387.

Washington M. and Zajac E. J. (2005). Status evolution and competition: theory and evidence. Academy of Management Journal, 48(2), pp. 282-296

Westphal, J. D. and Deephouse, D. L. (2011). Avoiding Bad Press: Interpersonal Influence in Relations Between CEOs and Journalists and the Consequences for Press Reporting About Firms and Their Leadership. Organization Science, 22(4), pp. 1061-1086.

Wolfe, A. W., \& Blithe, S. J. (2015). Managing image in a core-stigmatized organization: Concealment and revelation in Nevada's legal brothels. Management Communication Quarterly, 29(4), 539-563. 
Zavyalova, A., Pfarrer, M. D., \& Reger, R. K. (2017). Celebrity and infamy? The consequences of media narratives about organizational identity. Academy of Management Review, 42(3), 461-480.

Zavyalova, A., Pfarrer, M. D., Reger, R. K., \& Hubbard, T. D. (2016). Reputation as a benefit and a burden? How stakeholders' organizational identification affects the role of reputation following a negative event. Academy of Management Journal, 59(1): 253-276. 
Thomas Roulet est Senior Lecturer en Théorie des Organisations à l'Université de Cambridge, Judge Business School. Il est aussi chercheur associé à la business school de l'Université d'Oxford. Son travail porte sur les évaluations sociales, en particulier négatives, à différents niveaux d'analyse, ce qui l'amène à étudier les questions de réputation organisationnelle, scandales, et la marginalisation des minorités. Il est le co-éditeur en chef de M@n@gement, la première revue open-access dans le champ de la stratégie et de la théorie des organisations. 